\title{
The Neurobiology of Reference-Dependent Value Computation
}

\author{
Benedetto De Martino, ${ }^{1,2}$ Dharshan Kumaran, ${ }^{2}$ Beatrice Holt, ${ }^{2}$ and Raymond J. Dolan ${ }^{2}$ \\ ${ }^{1}$ California Institute of Technology, Pasadena, California 91125, and ${ }^{2}$ Wellcome Trust Centre for Neuroimaging, University College of London, London \\ WC1N 3BG, United Kingdom
}

\begin{abstract}
A key focus of current research in neuroeconomics concerns how the human brain computes value. Although, value has generally been viewed as an absolute measure (e.g., expected value, reward magnitude), much evidence suggests that value is more often computed with respect to a changing reference point, rather than in isolation. Here, we present the results of a study aimed to dissociate brain regions involved in reference-independent (i.e., "absolute") value computations, from those involved in value computations relative to a reference point. During functional magnetic resonance imaging, subjects acted as buyers and sellers during a market exchange of lottery tickets. At a behavioral level, we demonstrate that subjects systematically accorded a higher value to objects they owned relative to those they did not, an effect that results from a shift in reference point (i.e., status quo bias or endowment effect). Our results show that activity in orbitofrontal cortex and dorsal striatum track parameters such as the expected value of lottery tickets indicating the computation of reference-independent value. In contrast, activity in ventral striatum indexed the degree to which stated prices, at a within-subjects and between-subjects level, were distorted with respect to a reference point. The findings speak to the neurobiological underpinnings of reference dependency during real market value computations.
\end{abstract}

\section{Introduction}

Robert Parker, one of the world's leading wine critics, very often compares bottles of the same grape's variety produced in the same region within the same tasting session (Parker, 2002). The reason why Mr. Parker does not assign his score in isolation is because humans find it hard to compute values in the absence of a set reference point. Prospect theory elegantly captures this aspect of human behavior, introducing the notion of reference dependency to explain apparent inconsistencies across choices (Kahneman and Tversky, 1979). According to this view, people attribute value as a change from a set reference point, the perception of which is highly malleable. A paradigmatic example of reference dependent computation of value, that has a dramatic impact on financial transactions within real markets, is the endowment effect. This phenomenon refers to an observation whereby subjects value a good they own substantially more than an identical good that is available for purchase.

In a seminal experiment, subjects designated as sellers accepted a minimum of $\$ 7.00$ (willingness to accept as compensation or WTA) to part with a mug they had been given, whereas those designated as buyers were only willing to pay a maximum of $\$ 2.00$ (willingness to pay or WTP) (Kahneman et al., 1990). This

Received 0ct. 7, 2008; revised Nov. 26, 2008; accepted Dec. 22, 2008.

This work was supported by a Wellcome Trust Programme Grant to R.J.D. and the Wellcome Trust Fellowship to B.D.M. We thank P. Bossaerts, A. Rangel, H. Spiers, J. Kilner, and J. Hughes for their helpful discussions during the analysis of the study and their useful comments on this manuscript.

This article is freely available online through the J Neurosci Open Choice option.

Correspondence should be addressed to Benedetto De Martino, California Institute of Technology, 1200 East California Boulevard, MC 228-77, Pasadena, CA 91125. E-mail: bmartino@caltech.edu and b.martino@ucl.ac.uk. DOI:10.1523/JNEUROSCI.4832-08.2009

Copyright $\odot 2009$ Society for Neuroscience $\quad 0270-6474 / 09 / 293833-10 \$ 15.00 / 0$
WTA-WTP discrepancy is not explicable under the assumption that people have a stable and absolute representation of value. Furthermore, this behavior strikingly contradicts a prediction of classical economic theory that stipulates that the price assigned to an object, during a costless market transaction, should reflect solely the value of the item (Willig, 1976) and be independent of a subject's role (i.e., as buyer or seller) in the transaction. In contrast, this apparently inconsistent behavior can be resolved by considering that the value of a good (or an option) is computed with respect to a reference point corresponding to ownership of the item, which can be considered the status quo (Kahneman et al., 1991). For this reason buyers evaluate the purchase of an object as a potential gain whereas sellers view the transaction as a potential loss, demanding a higher price as of loss aversion (Kahneman and Tversky, 1979; Kahneman and Tversky, 2000).

In this experiment we probed the types of neural computations involved in the generation of reference-dependent values during actual market transactions. To achieve this, we combined a functional magnetic resonance imaging (fMRI) parametric design with a novel economic exchange task in which subjects appraise the value of "goods" from the perspective of either a buyer or a seller (Fig. 1). Given that subjects were asked to evaluate

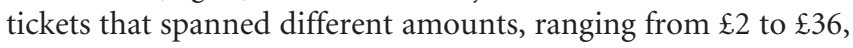
we were able to identify brain regions performing referenceindependent computations of value, as investigated by previous studies (Plassmann et al., 2007). Critically, however, our paradigm was optimized to identify the neural circuitry supporting reference-dependent value computations, thus allowing us to ask which brain regions track the price discrepancy observed between buying and selling (i.e., WTP $<$ WTA; endowment effect).

Our results reveal a striking dissociation between brain re- 
gions supporting reference-independent, and reference-dependent computations. As such, neural activity in both OFC and dorsal striatum tracked subjects' actual stated prices, during buying and selling. In contrast, activity in ventral striatum indexed the degree to which subjects' stated prices were distorted with respect to a reference point, which was shifted depending on the type of transaction (i.e., buying vs selling).

\section{Materials and Methods Subjects}

Twenty-five subjects participated in the study. All subjects had a university degree, or were in the process of obtaining one. A highly significant behavioral endowment effect (i.e., WTA $>$ WTP $)\left(p<0.0001 t_{(17)}=5.02\right.$ two-tailed paired $t$ test) was observed across the entire group ( $n=25)$ who participated in the scanning phase of the experiment, confirming the robustness of our experimental protocol in eliciting a reliable endowment effect. A group of 18 subjects [10 males (mean age 22.2 years \pm 3.1 ) and 8 females (mean age 24.6 years \pm 3.5 )] were included in the final imaging analysis, which was designed specifically to reveal brain regions mediating the endowment effect. One subject was excluded as of excessive head movement in the scanner. Six subjects, who pursued a deviant strategy with no theoretical basis in the literature (WTA > WTP: significant reverse endowment effect, $p<0.01$ ), were excluded after two sessions. Evidence obtained from debriefing these individuals indicated that their pattern of behavior resulted from a misconstrual of the experimental goals as maximization of the number of successful market transactions effected by the BDM mechanism, rather than pursuing an overall financial gain. The study was conducted with the approval of the $\mathrm{Na}$ tional Hospital for Neurology and Neurosurgery and the Institute of Neurology Joint Research Ethics Committee.

\section{Experimental paradigm}

The subjects were instructed carefully on all aspects of the experiment to ensure that they clearly understood the nature of the task. To enhance clarity, we used a detailed computer tutorial. Finally, subjects were required to correctly complete a questionnaire before proceeding to the scanning phase to ensure that instructions were completely understood by the subject. The paradigm was divided into three phases: endowment, scanning, and transaction phases, respectively.

\section{Endowment phase}

Before scanning, each subject was asked to choose either of two distinct decks (red or green) each containing 18 lottery tickets. Subjects were endowed (i.e., given) with the selected deck (e.g., red) and instructed that they would have the opportunity during scanning to buy tickets belonging to the other deck. Subjects were also endowed with an amount of cash (£36) with which to buy tickets.

Each deck was composed of 18 tickets, each having a different value, highlighted on the front, ranging between $£ 2$ and $£ 36$ in increments of $£ 2$. On the back of each ticket was written, hidden by a black scratchable covering, the actual amount that the ticket was worth (in pence and pounds). Subjects were told that this amount was determined randomly by a computer program, and therefore equally likely to be any figure

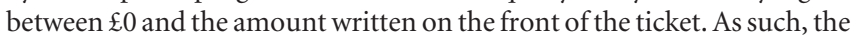
expected value of the ticket was one half of the amount written on the front of the ticket.

\section{Scanning phase}

The scanning phase was divided in four sessions of 12 min each. During each trial, a screen displayed one of three tickets: (1) a ticket from the deck subjects had selected (e.g., red ticket: selling transaction), (2) a ticket from deck they did not select (e.g., green ticket: buying transaction), or (3) a ticket from a third deck (yellow: evaluate condition) (Fig. 1).

Five different experimental conditions were used, and presented in pseudorandom order.

Subject's selling transaction (You_SELL). The screen displayed one of the subject's own tickets (i.e., taken from the deck they had selected before scanning). By moving a cursor on a bar subjects were asked to state the minimum selling price (WTA) that they would be happy to accept during the transaction phase, prices ranging between zero and the value indicated on the front of the ticket. 
Subject's buying transaction. The screen displayed a ticket from the deck that the subject did not select before scanning (e.g., green). By moving a cursor on a bar subjects were required to state the maximum buying price (WTP) that they would accept to pay during the transaction phase. Possible prices ranged between zero and the value indicated on the front of the ticket.

Subject's evaluate condition. The screen displayed a ticket from a third deck (i.e., yellow) By moving a cursor on a bar subjects were asked to state the amount they felt the ticket was worth (i.e., evaluation price). The yellow deck was not used in the transaction phase. Possible prices ranged between zero and the value indicated on the front of the ticket.

Computer's selling transaction. The screen displayed one of the subject's own tickets (i.e., taken from the deck they had selected before scanning). The computer produced a minimum selling price for that ticket by showing a box on the price bar. The price was randomly generated such that it was $>0.5 \times \mathrm{EV}$ and $<1.5 \times \mathrm{EV}(\mathrm{EV}=$ expected value of the ticket) and represented by a box positioned on the price bar. The subject was required to accept this price and was required to move the cursor in the box with the computer price.

Computer's buying transaction. The screen displayed a ticket from the deck that the subject did not select before scanning (e.g., green). The computer produced a maximum buying price for that ticket by showing a box on the price bar. The subject was required to move the cursor to within the position of the box. The price selected by the computer was determined as described above. The subject was required to accept this price and was required to move the cursor in the box with the computer price.

\section{Transaction phase}

At the end of the scanning phase one of the four sessions was randomly selected and one ticket for selling, and one for buying, was extracted from this session. These two tickets were used to perform a real money transaction using the Becker-DeGroot-Marschak (BDM) mechanism (Becker et al., 1964). The BDM mechanism is widely used in experimental economics as an incentive-compatible procedure for eliciting nonstrategic reservation prices.

Selling transaction. One of the selling tickets was randomly extracted for the transaction (e.g., the ticket with $£ 20$ written on the front). A single ball was extracted from a bingo cage which contained a quantity of balls numbered from 1 to $n$ (in increments of 1 ; where $n=$ value written on the ticket). The value on the ball extracted from the cage yielded the BDM buying price in pounds (e.g., $\mathfrak{1 2}$ ). The amount in pence was determined by a roll of dice (e.g., £12.30). If the subject's (or the computer's) minimum selling price (WTA: e.g., £8) for that ticket was lower than the BDM price (e.g., $\mathfrak{E} 12.30$ ) the subject received the BDM buying price (e.g., $£ 12.30$ ) and sold the ticket. Alternatively, if the subject's price (e.g., $£ 14.60)$ was higher than the BDM price the transaction did not go through and the subject kept his/her ticket. In this case she/he was allowed to scratch the back of her/his ticket and receive in cash the amount written on the back of the ticket.

Buying ticket transaction. One of the buying tickets was randomly extracted for the transaction (e.g., the ticket with $£ 8$ written on the front). A ball was extracted from a bingo cage which contained a quantity of balls numbered from 1 to $n$ (in increments of 1 ; where $n=$ value written on the ticket). The value on the ball extracted from the cage yielded the BDM buying price in pounds (e.g., $\mathfrak{E} 5$ ). The amount in pence was determined

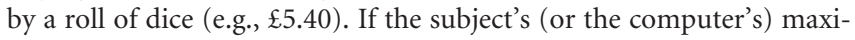

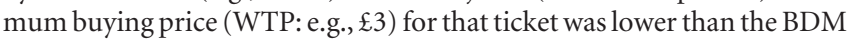
selling price (e.g., $\mathfrak{E} 5.40$ ) the transaction did not go through and the subject kept his/her money without receiving the ticket. Otherwise if the

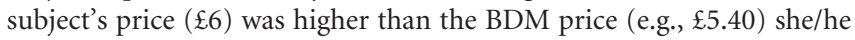

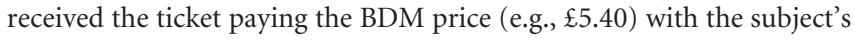
amount cash received at beginning. In this second case, the subject was allowed to scratch the back of her/his ticket receiving in cash the winning amount of that ticket.

Subjects were given their total winnings at the end of the experiment. This was derived from (1) cash remaining from the initial endowment they received at the start of the experiment (£36), (2) cash gained from a selling transaction going through (at a price determined by the BDM), and (3) cash gained from receiving the amount written on the back of a ticket (that they had either acquired through a buying transaction, or not sold).

\section{Behavioral data analysis}

Behavioral data were analyzed using the statistic software SPSS. We calculated, for each subject, the WTA (selling price) and WTP (buying price) for each individual ticket averaging the subject's prices across the four scanning sessions. These results were collapsed across all subjects for a group level analysis. The behavioral endowment effect (WTA-WTP price discrepancies) were calculated using a two-tailed paired $t$ test. The evaluate condition (e.g., ticket that were not traded) was used to estimate the subjective EV (sub-EV) for each ticket and each subject. Again the difference between WTA/sub-EV and WTP/sub-EV was calculated, either at subject level and at group level, using a two-tailed paired $t$ test.

\section{Image acquisition and analysis}

Gradient-echo T2*-weighted images (EPI) were acquired on a 1.5 tesla magnetic resonance scanner using a $30^{\circ}$ tilted acquisition sequence designed to reduce signal dropout in orbitofrontal lobes. Image parameters were as follows: TE $50 \mathrm{~ms}$; TR $3.96 \mathrm{~s}$; slice thickness $2 \mathrm{~mm}$; interslice gap $1 \mathrm{~mm}$. We collected 648 volumes (across four sessions) per subject. T1weighted structural images coregistered with mean EPI images and averaged across subjects to allow group level anatomical localization. Images were analyzed using the statistical parametric software SMP2 (Wellcome Department of Imaging Neuroscience London, www.fil.ion.ucl.ac.uk/ spm). Preprocessing consisted of spatial realignment and normalization to a standard EPI template, and spatial smoothing ( $8 \mathrm{~mm}$ kernel). The fMRI data were analyzed in an event-related manner using the general linear model, using the SPM2 statistical analysis software. After discarding the first six image volumes from each run to allow for T1 equilibration effects, image volumes were realigned and coregistered to each subject's structural scan. Subject-specific regressors of interest were assembled by convolving $\delta$ functions (corresponding to the time of onset of the choice pair, for each condition) with a canonical hemodynamic response function (HRF). We removed low frequency fluctuations by a high-pass filter with a cutoff at $128 \mathrm{~s}$. A correction for temporal autocorrelation in the data (AR $1+$ white noise) was applied. Parameter estimates were used to calculate the appropriate linear contrast. These contrast images were then entered into a one-sample $t$ test across all subjects (random effects analysis).

The first analysis sought to isolate brain activity correlated with changes in WTP and WTA. We constructed a general linear model (GLM) with four main regressors coding for each condition (You_Buy; You_Sell; Computer_Buy; Computer_Sell). The onset of these regressors was time locked to the time when the initial message appeared on the screen. Four trial-by-trial parametric regressors were included for each condition separately (You_WTP; You_WTA; Computer_WTP; Computer_WTA) that were derived from the prices that subjects stated during each transaction. Finally we included six motion parameters and one regressor of no interest (coding for the evaluate condition). Experimental conditions were modeled with a boxcar function of $8.5 \mathrm{~s}$ (the entire time in which the condition was on the screen and the subjects were stating their prices).

The primary aim of our neuroimaging analyses was to identify brain regions mediating the endowment effect (Fig. 1). To highlight brain areas in which activity correlated with the magnitude of the price discrepancy in the buy and sell domains, we constructed a second GLM. As before, the four categorical regressors coded each condition separately (You_Buy; You_Sell; Computer_Buy; Computer_Sell). The critical difference was that in this second GLM the other parametric regressors used to modulate the categorical regressors did not encode the WTP and the WTA themselves. Instead, the parametric regressors encoded the percentage change in subjects' prices (or the computer's price) from the sub-EV for each specific ticket, in both the buying and selling domain (i.e., $\Delta$-WTP

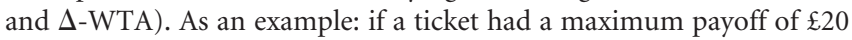
and the sub-EV was $\mathfrak{E} 10$ and subject WTP $=\mathfrak{E} 12$; then the percentage price deviation $(\Delta$-WTP) was coded as $+20 \%$. In contrast if the subject 
price $=\mathfrak{£} 8$ the percentage price deviation $(\Delta$-WTP) was coded as $-20 \%$. Finally, as in the first GLM, we included six motion parameters and the one regressor of no interest (the evaluate condition). Conditions were again modeled with a boxcar function of $8.5 \mathrm{~s}$.

In all statistical comparisons reported in this study, we identified regions specifically involved in the evaluation process, whose activity correlated with either subjects' stated prices themselves (i.e., WTA or WTP), or the respective percentage price deviations ( $\Delta$-WTA and $\Delta$-WTP), by subtracting out the relevant computer (i.e., control) condition. All the statistical contrasts calculated for each subject were subsequently taken to the random effects level to perform a group analysis in line with established procedures, by means of a one-sample $t$ test (Friston, 1995).

Furthermore we also performed a between subjects analysis as follows: for each subject the size of the behavioral WTA-WTP disparity was calculated by subtracting the mean selling prices from the mean buying prices for all the tickets traded during the experiment. A simple correlation analysis was performed to identify voxels in which activity in the endowment statistical contrast [(You_Sell-You_Buy) - (Computer_Sells-Computer_Buys)] for a given subject directly correlated with the size of WTA-WTP disparity across the entire experiment.

We report results in a priori regions of interest (striatum, insula, and orbital and medial prefrontal cortex) motivated by the fact that these are regions typically identified in neuroimaging studies financial gain evaluation (O’Doherty, 2004; Nieuwenhuis et al., 2005; Seymour et al., 2007; Tom et al., 2007) at $p<0.001$ uncorrected for multiple comparisons $(Z<3.1$ ) (unless otherwise stated) and/or at $p<0.05$ small volume correction for multiple comparison (SVC) using a $8 \mathrm{~mm}$ sphere centered on the peak activity for the a priori regions of interest as reported by previous studies. Activations in other regions are reported if they survive whole-brain correction for multiple comparisons at $p<0.05$. For display purposes in this study, statistic images are shown with $Z>2.6$ corresponding to $p<0.005$. Finally we reported the size of each cluster in number voxels surviving the statistical threshold of $Z>2.6$.

\section{Results}

Twenty-five subjects participated in the study, 18 of whom [10 males (mean age 22.2 years \pm 3.1 ) 8 females (mean age 24.6 years \pm 3.5$)$ ] were included in the final analysis (see methods). Before fMRI scanning participants received a deck of lottery tickets whose maximum payoff ranged from $\mathfrak{2} 2$ to $\mathfrak{E} 36$, as well as a cash sum (£36). The fMRI experiment consisted of five pseudorandomly presented conditions. "You_Sell” required subjects to assign a minimum selling price (WTA) for their own lottery tickets. "You_Buy" required subjects to assign a maximum buying price (WTP) for another deck of lottery tickets. "Computer_Sell" and "Computer_Buy" conditions involved a computer randomly selecting a maximum selling or buying price which subjects were passively required to accept. Additionally, a fifth condition "You_Evaluate" required subjects to assign a value to a given ticket without an ensuing transaction. This last condition provided a means to estimate, for each participant, the subjective expected value (sub-EV) of a given ticket independent of a subjects' position in a transaction. Finally, at the end of scanning, one sell and one buy trial (either "You" or "Computer") were randomly selected and used to perform an actual economic transaction using the Becker-DeGroot-Marschak (BDM) incentivecompatible scheme (Becker et al., 1964) (Plassmann et al., 2007) (see Materials and Methods for more details). At the end of the experiment, subjects received a real cash payment proportional to their total winnings calculated by adding together the amount of cash remaining from the initial endowment, cash earned through the sale of a ticket, and the amount concealed on the back of tickets remaining in their possession.

\section{Behavioral data}

Critically, we elicited a robust endowment effect reflecting a shift in reference point arising out of a subjects' position as buyer or seller in the market transaction, rather than arising from how options themselves are framed, as studied previously (De Martino et al., 2006). At the behavioral level, our findings replicate between-subjects studies which have shown that there is a systematic increase in the minimum selling price (WTA) (Fig. 2, red line), compared with the maximum buying price (WTP) (Fig. 2, green line) for a ticket (or object) with the same expected payoff $\left(p<0.0001 t_{(17)}=7.47\right.$ two-tailed paired $t$ test $)$. Importantly, in the evaluate condition, which did not involve any transactions, subjects assigned ticket prices (Fig. 2, yellow line) that fell between the overall average selling and buying prices, being significantly different from both (buy-evaluate: $p<0.0001 t_{(17)}=9.57$; sell-evaluate: $p<0.0001 t_{(17)}=5.61$; two-tailed paired $t$ test).

\section{Imaging data}

Subjects performed the task while being scanned using fMRI, a situation that enabled us to obtain on-line measures of regional brain activity. Our experimental design enabled us to isolate brain activity associated with the prices subjects judged tickets to be worth, for buying (i.e., WTP) and selling (i.e., WTA). For each condition separately (You_WTP; You_WTA; Computer_WTP; Computer_WTA), we constructed a vector derived from the price subjects judged a ticket to be worth on each trial. These vectors were then convolved with the hemodynamic response function (HRF) to create parametric regressors of interest. Statistical analysis was performed using the general linear model (GLM) according to standard procedures (Friston, 1995) (see detailed Materials and Methods). By performing a linear contrast over these parametric regressors, we were able to identify brain regions exhibited a significant correlation between neural activity and subjects' stated ticket price (i.e., WTA, or WTP). Importantly, these contrasts were performed relative to the computer condition (e.g., You_WTP vs Computer_WTP), so as to isolate brain regions specifically associated with a value computation rather than other aspects of the task (e.g., stimulus viewing). Of note, the computer conditions (WTP and WTA) were identical in all aspects to the You conditions with the critical difference being that subjects' stated prices resulted from an active evaluation process in the You conditions, but were determined by a cursor in the Computer conditions (see Materials and Methods).

We initially performed a whole brain SPM analysis to identify brain regions in which neural activity reflected the price subjects were willing to pay for lottery tickets (i.e., WTP) (see Materials and Methods). Activity in medial orbitofrontal cortex (m-OFC: activity peak: $\left[x=-4, y=40, z=-16 Z_{\text {(score) }}=4.27 ; 35\right.$ voxels $]$ $p<0.001$ uncorrected and $p<0.05$ SVC) showed a significant correlation with the WTP (Fig. $3 a$ ), a finding consistent with a recent study showing that activity in the same area (m-OFC) codes for the WTP during the purchase of food by hungry subjects (Plassmann et al., 2007). In contrast, activity in a more lateral portion of OFC correlated with the price subjects assigned to tickets during selling (WTA) (activity peak: $[x=-36, y=42, z=$ $-10 Z_{\text {(score) }}=3.33 ; 24$ voxels $] p<0.001$ uncorrected and $p<$ 0.05 SVC) (Fig. 3b).

Although activity in these regions ( $\mathrm{m}-\mathrm{OFC}$ and l-OFC) correlated with subjects' stated prices either during selling or buying respectively, activity in dorsal striatum alone exhibited an increase in activity during both selling and buying (i.e., WTA and WTP) (activity peak: $\left[x=8, y=12, z=12 Z_{\text {(score) }}=3.69 ; 79\right.$ voxels] $p<0.001$ uncorrected and $p<0.05$ SVC) (Fig. $3 c$ ). Ac- 

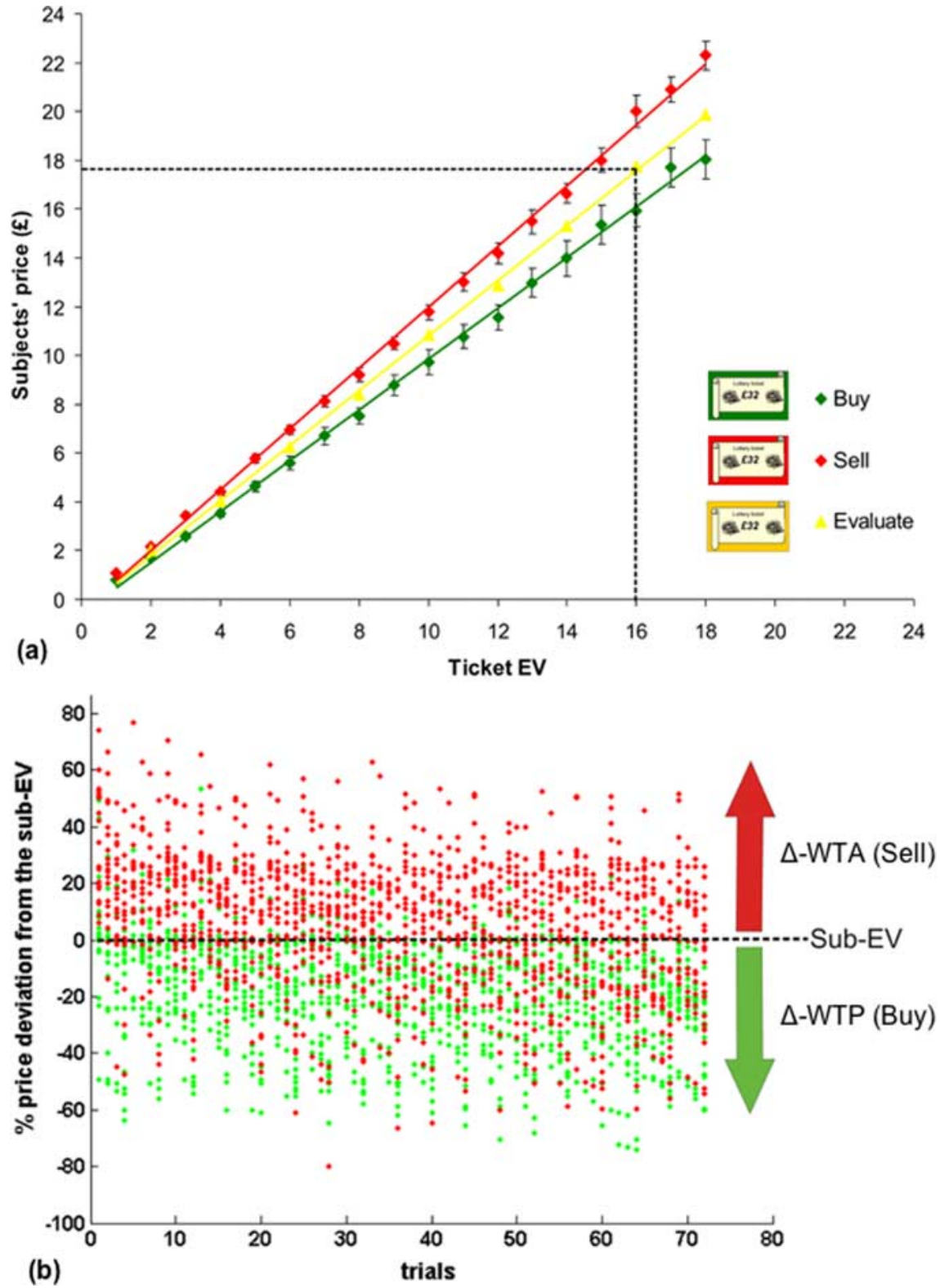

Figure 2. Behavioral results. $\boldsymbol{a}$, This graph shows the prices assigned to each ticket during the scanning phase, averaged across all subjects $(n=18)$. The ticket expected value $(\mathrm{EV})$ is represented on the $x$-axis and the subjects' prices are on the $y$-axis. The minimum buying prices (WTP, green line) are significantly lower than the maximum selling price (WTA, red line) (see Results). The yellow line represents the evaluation prices that were used to calculate the subjective ticket $\mathrm{EV}$ (sub-EV). For display purposes a dotted line represents the prices for a ticket with an $\mathrm{EV}$ of $£ 16$. Note that in the figure the buying tickets are represented in green and the selling tickets in red; in the actual experiment each subject selected a ticket color during the endowment phase. $\boldsymbol{b}$, This graph represents the trial-by-trial ( $x$-axis) percentage $(\%)$ deviation from the sub-EV for a subject's stated selling price on a given trial: ( $\Delta$-WTA red dots) and a subject's stated buying price on a given trial: ( $\Delta$-WTP green dots) tickets ( $y$-axis). The two arrows represent the within-subjects fMRI analysis that identifies areas correlated with an increase in $\Delta$-WTA price and a decrease in $\Delta$-WTP.

tivity in this region was statistically significant in each condition independently: WTA (activity peaks $[x=-10, y=22, z=6$ $Z_{\text {(score) }}=3.15 ; 31$ voxels $] p<0.001$ uncorrected and $p<0.05$ SVC and $\left[x=8, y=14, z=12 Z_{(\text {score })}=2.01 ; 2\right.$ voxels $] p<0.005$ uncorrected and $p<0.05$ SVC) and WTP (activity peak $[x=$ $-12, y=14, z=4 Z_{\text {(score) }}=3.39 ; 9$ voxels $] p<0.001$ uncorrected and $p<0.05$ SVC. As WTA and WTP are in fact highly correlated with the actual ticket EV a similar pattern of correlation was found when we used the ticket EV, rather than the subjects' WTA and WTP, as parametric regressors. Our findings show that when subjects assign a higher price to a ticket, during either buying or selling, activity in OFC and dorsal striatum is greater. Indeed, neural activity in these regions also tracked the expected value of lottery tickets (e.g., $\mathfrak{E} 1$ to $\mathfrak{E} 18$ ), suggesting that these brain areas code value in an absolute manner (i.e., independent of a reference point).

Our critical neuroimaging analysis, however, sought to identify brain areas in which neural activity correlated with the magnitude of shift in value between the buy and sell domains in accord with the behavioral effect we elicited (i.e., endowment effect), a parameter of a reference dependent valuation. Importantly, subjects exhibited a high degree of variability in the prices assigned to each ticket such that the magnitude of the percentage deviation of the WTA or WTP from the sub-EV (i.e., $\Delta$-WTA and $\Delta$-WTP) showed trial-to-trial fluctuations (Fig. 2b). We therefore included four regressors $(\Delta$ WTA and $\Delta$-WTP for both You and Computer) in our general linear model, coding for trial-by-trial percentage price deviations from the respective sub-EV (calculated on the basis of the evaluation tickets). Notably, although the real expected ticket value (ticket-EV) was usually close to sub-EV we used the latter to calculate the percentage deviation seen for selling or buying prices. Indeed, using the sub-EV, rather than the ticket-EV, is advantageous as it controls for a well described distortion of outcome probability and general risk attitude (Kahneman and Tversky, 1979).

We reasoned that a brain region that plays a central role in mediating the endowment effect (i.e., the WTA-WTP disparity) should exhibit greater activity on a given trial when a subject assigns a higher price in the sell domain (i.e., increase in $\Delta$-WTA), or a lower price in the buy domain (i.e., decrease $\Delta$-WTP). Hence, our critical contrasts sought to identify brain areas in which activity followed this prescribed pattern in our trial-by-trial parametric analysis (Fig. 2b). Note that to isolate brain regions specifically associated with a value computation arising from a shift in reference point we again subtracted out nonspecific activity correlated with increasing selling and decreasing buying prices in the computer condition.

Bilateral ventral striatum showed a pattern of activity consistent with the behavioral effect elicited by our task (i.e., WTAWTP disparity), positively correlating with an increase in selling prices (i.e., increase in $\Delta$-WTA) and a reduction in buying prices (i.e., decrease in $\Delta$-WTP), an effect expressed solely when subjects themselves were agents in the evaluation process (i.e., when activity from the computer conditions was subtracted out). Importantly, activation in OFC did not correlate, even at a liberal 
threshold of $p<0.01$ uncorrected, with the $\Delta$-WTP or $\Delta$-WTA, in line with the conclusion that this region implements reference-independent computations (see above). By examining correlations in the interaction contrast [ $\Delta$-WTA_You $\Delta$-WTP_You $)-[(\Delta$-WTA_Computer $\Delta$-WTP_Computer)] we confirmed ventral striatal activity was not driven selectively by either the buy or sell condition, but by both conditions together (activity peaks: $\left[x=26, y=14, z=0 Z_{\text {(score) }}=3.56\right.$ 62 voxels $] p<0.001$ uncorrected and $p<$ $0.05 \operatorname{SVC}[x=-28, y=-6, z=-4$ $Z_{\text {(score) }}=2.9435$ voxels $] ;[x=-16, y=6$, $z=8 Z_{\text {(score) }}=2.8614$ voxels] $p<0.005$ uncorrected and $p<0.05$ SVC) (Fig. 4). This region, therefore, correlated significantly with increasing prices in the selling condition (i.e., increase in $\Delta$-WTA) (activity peak: $\left[x=26, y=14, z=0 Z_{\text {(score) }}=\right.$ 3.5726 voxels] $p<0.001$ uncorrected and $p<0.05$ SVC), and decreasing prices in the buying condition (i.e., decrease in $\Delta$-WTP) (activity peak: $[x=24, y=20$, $z=-6 Z_{\text {(score) }}=3.3615$ voxels $] p<0.001$ uncorrected and $p<0.05$ SVC). Importantly, activity in the ventral striatum, in contrast with dorsal striatum, did not correlate, even at a liberal threshold of $p<$ 0.01 uncorrected, either with the WTP or WTA nor with the ticket-EV. These data, therefore, indicate that the ventral striatum implements a value computation that is indexed relative to a reference point (namely the deviation from the sub-EV), contingent on a subject's position in the economic transaction, rather coding for the ticket value per se (i.e., the WTP/WTA or ticket-EV).

We also identified increased bilateral anterior insula activity in this key interaction contrast (Fig. 4c): right insula (activity peaks: $\left[x=50, y=12, z=-6 Z_{\text {(score) }}=3.07168\right.$ voxels $] p<$ 0.005 uncorrected and $p<0.05$ SVC) and left insula $[x=-54$, $y=20, z=-2 Z_{\text {(score) }}=3.69168$ voxels $] p<0.001$ uncorrected and $p<0.05$ SVC). In contrast to the ventral striatum, neural activity in insula correlated only with decreasing prices in the buying condition (i.e., decrease in $\Delta$-WTP) and not with increasing prices in the selling condition (i.e., increase in $\Delta$-WTA). The variant pattern of activity exhibited by ventral striatum and insula suggests that these two regions compute different types of signals which may reflect a reward prediction error signal (PE) and risk prediction error signal ( $r-P E)$, respectively (see Discussion).

We next reasoned that if the striatum plays a role in tracking the changes in value according with the subject's role in the transaction (i.e., WTP and WTA price discrepancy), then subjects who showed a larger WTA-WTP discrepancy, over the course of the experiment (i.e., larger endowment effect), should exhibit a tighter coupling between trial-by-trial striatal activity and price discrepancy. To assess this, we performed a between-subjects analysis using the statistical contrast described previously $[(\Delta-$ WTA_You - $\Delta$-WTP_You $)-[(\Delta$-WTA_Computer $\Delta$-WTP_Computer)]. Using each subject's overall susceptibility
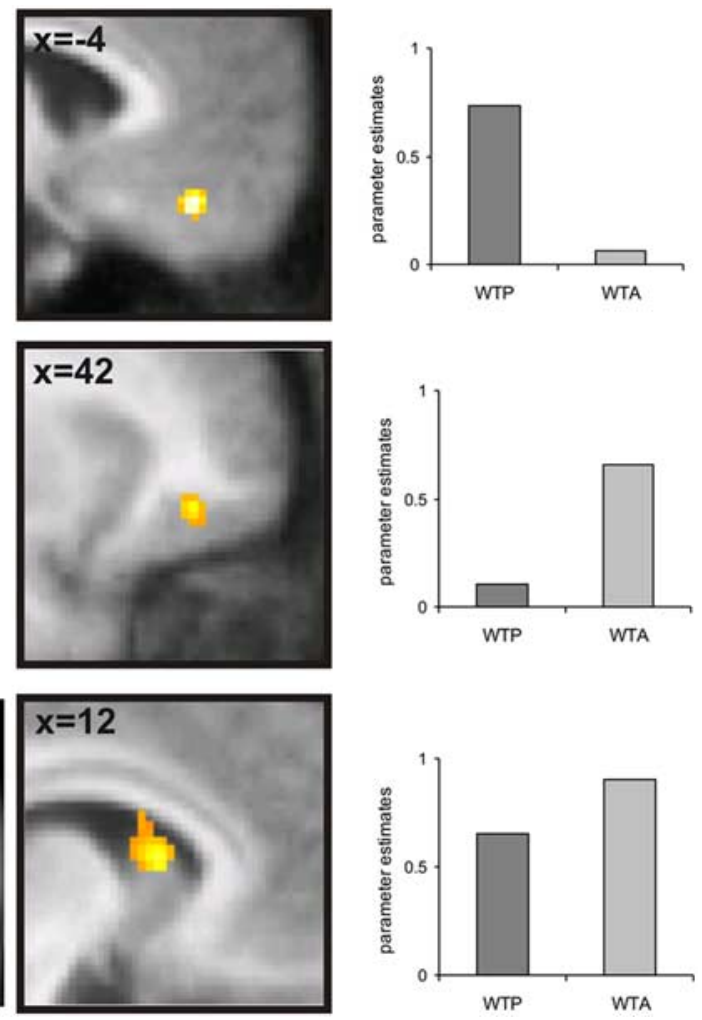

Figure 3. fMRI results: Parametric correlation with WTA and WTP. a shows a positive correlation between WTP and activity in condition (i.e., WTP and WTA) subtracted for the baseline conditions (i.e., forced computer conditions, see Materials and Methods for more details). Note that no error bars are displayed because reported statistical comparisons reflect within-subject effects. Effects are shown at $p<0.005$ for display purposes.

to the endowment manipulation as a between subject statistical regressor, we observed a significant correlation in ventral striatum with this measure: [left: $x=-14, y=10, z=-6 Z_{\text {(score) }}=$ 3.88116 voxels] [right: $x=18, y=4, z=-6 Z_{\text {(score) }}=2.94$ ] $p<$ 0.005 uncorrected and $p<0.05$ SVC; 5 voxels; [right: $x=18, y=$ $4, z=10 Z_{\text {(score) }}=3.04 ; 55$ voxels $] p<0.001$ uncorrected and $p<$ 0.05 SVC) (Fig. 5). Thus, activity in ventral striatum not only tracked a price discrepancy on a trial-by-trial basis but also predicted on individual subjects' basis the magnitude of behavioral shift arising from the manipulation of the reference point during the experiment.

\section{Discussion}

In this study we used fMRI and an economic exchange paradigm to dissociate between brain regions in which activity reflected a reference independent computation (i.e., increase in WTA, WTP), as opposed to a reference dependent value computation (i.e., correlation with $\Delta$-WTA, $\Delta$-WTP). We found that whereas activity in OFC and dorsal striatum was positively correlated with subjects' stated price, and also ticket-EV, activity in ventral striatum indexed price distortions during buying $(\Delta-\mathrm{WTP})$ and selling $(\Delta$-WTA). Our results reveal a neural correlate of referencedependent value computation, which suggests a specific role for the ventral striatum in computing value relative to a reference point, a key variable in the generation of the endowment effect.

One interesting finding in our data was that neural activity in OFC tracks the incentive value of lottery tickets. This result is in 
(a)
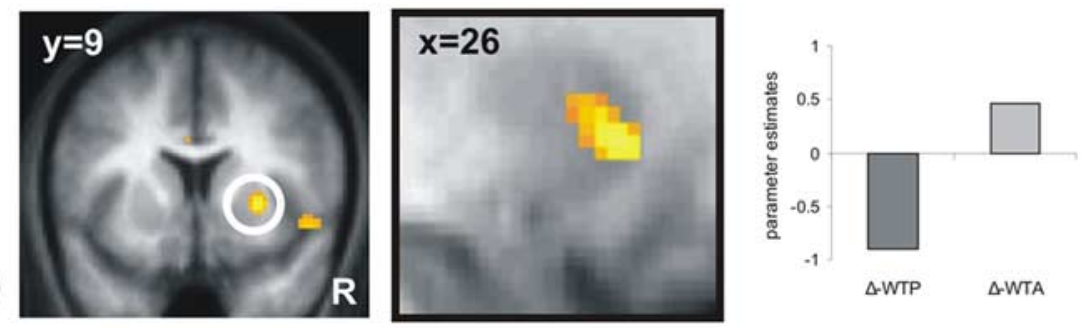

(b)
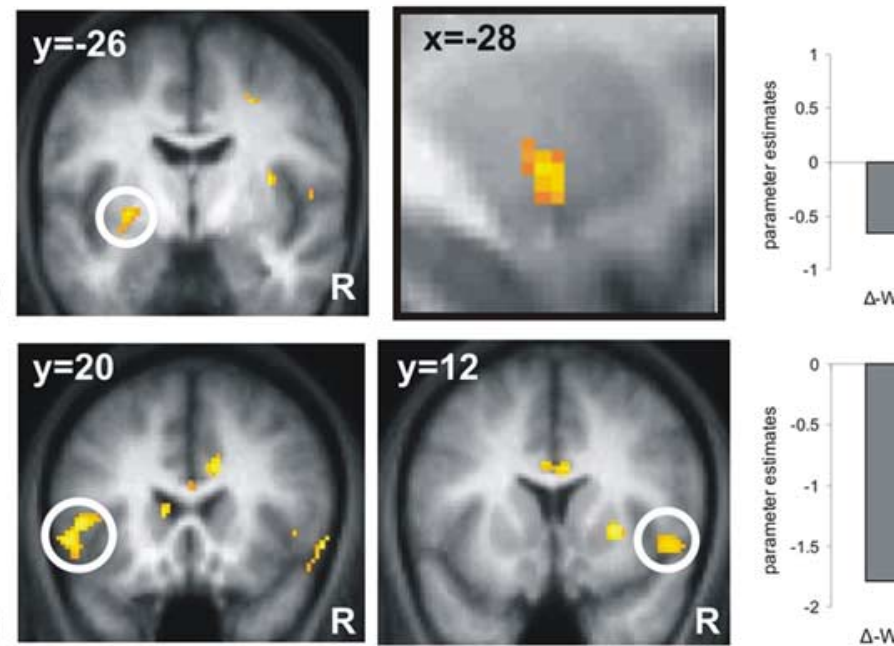

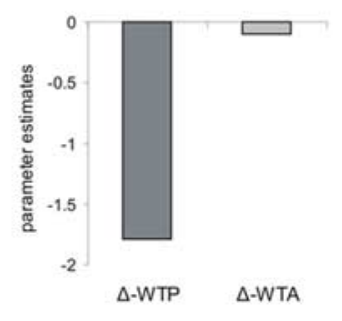

suggests that transactions are perceived as a potential loss from the perspective of the seller.

An interesting question concerns the role of the OFC in reference-dependent value computation. A recent study found that a value signal encoded by neurons in monkeys' OFC is invariant for changes in the choice menu (Padoa-Schioppa and Assad, 2008). In other words, the representation of value encoded by this neuron population is stable and not relative to the other available options. Although our findings clearly support this hypothesis, it should be noted that other studies (Tremblay and Schultz, 1999; Elliott et al., 2008) report OFC neurons encode the value of an option relative to available alternative choices, rather than in absolute manner.

In our experiment, neural activity in dorsal striatum (i.e., caudate nucleus), like the OFC, tracked both the actual price subjects assigned to tickets (WTP, WTA), and ticket-EV, measures that are in fact highly correlated. In contrast to the OFC, activity in a single region of dorsal striatum mirrored subjects' prices during both buying and selling, suggesting a more generic role in reference-independent value computation. Activity in the dorsal striatum has been found to be responsive to the presence, and magnitude, of expected future reward (O'Doherty et al., 2002; Cromwell and Schultz, 2003; Delgado et al., 2003), particularly in situations in which the reward is contingent on the execution of an action (O'Doherty et al., 2004; Tricomi et al., 2004). In our study, activity specifically represented subjects'

line with evidence that neurons in the OFC encode the incentive value of the stimuli at the time of decision making (Wallis and Miller, 2003; Roesch and Olson, 2004; Padoa-Schioppa and Assad, 2006; Wallis, 2007; Rolls et al., 2008), and perform reference-independent value computations (e.g., expected value of option). For instance, a recent fMRI study (Plassmann et al., 2007) reported that BOLD activity in the medial OFC shows a positive correlation with the price hungry subjects were willing to pay for items of food (WTP). Our findings not only replicate this earlier finding in relation to a primary reinforcer (Plassmann et al., 2007), but also provide evidence that dissociable regions of the OFC (i.e., medial for WTP and lateral for WTA) play a role in buying and selling for secondary reinforcers such as money. We note, a recent report (Hare et al., 2008) showing that although activity in $\mathrm{m}$-OFC correlates with the WTP, it does not reflect the actual item price. Conversely activity in l-OFC in this study (Hare et al., 2008) was found to track the actual price of the item. An interpretation of our findings that would reconcile these latter findings is that subjects may be engaged in a computation of loss during WTA elicitation, reflected in a price-like signal in l-OFC (Hare et al. 2008), rather than a cost-benefit trade-off signal apparent in $\mathrm{m}$-OFC during WTP elicitation. Indeed, this interpretation of our data dovetails with a psychological framework within Prospect Theory (Kahneman and Tversky, 1979), which stated price, during active evaluation (You conditions) as opposed to passive evaluation (Computer conditions), in both buying (WTP) and selling (WTA) conditions. Our results, therefore, support the view that the dorsal striatum participates in value computations primarily when this computation is directly relevant to action selection (Balleine et al., 2007).

Although our findings provide evidence that brain regions such as the OFC and dorsal striatum encode microeconomic parameters (e.g., EV) by computing reference-independent value, the primary aim of our study was to characterize the neural underpinnings of the computation of reference-dependent value. At a behavioral level, we demonstrated a robust endowment effect (i.e., WTP $<$ WTA), whereby subjects assigned significantly different values to identical options (i.e., lottery tickets), depending on the reference point from which they viewed the transaction (i.e., as owner or purchase of the ticket). Critically, activity in ventral striatum, during both buying and selling, tracked on a trial-by-trial basis the magnitude to which a subject's stated price deviated from the price assigned during "neutral" conditions (i.e., evaluate condition: sub-EV). Strikingly, activity within ventral striatum predicted price deviations (i.e., from sub-EV) within a subject on a trial-by-trial basis, but also explained a significant degree of between-subject variability in the size of endowment effect measured. 
Several recent neuroimaging studies using financial decision making tasks provide evidence that the human striatum computes several microeconomics parameters of anticipated gains (e.g., expected value, magnitude and probability of a gamble) (Yacubian et al., 2006; Tobler et al., 2007). Here, we show that activity in ventral striatum reflects a computation of value indexed relative to a reference point (namely the deviation from the sub-EV), which changes according to the role of the subject in the transaction (i.e., buyer vs seller). Market transactions, in contrast to experimental settings in which a given stimulus predicts an expected gain, require neural computations of value to incorporate a trade-off between the cost of the item and its expected value, rather than simply the magnitude of an expected gain. As such, our findings indicate that during market exchanges this value computation is dynamically configured with respect to a subjects' role as a buyer or seller as predicted by prospect theory (Kahneman and Tversky, 1979), perhaps indicating that activity in ventral striatum may reflect a psychological tendency toward loss aversion (Seymour et al., 2007).

A key issue concerns the exact neural mechanisms underpinning the computation of reference-dependent value in the ventral striatum. Although our data cannot provide a definite answer to this question, it is important to note that the pattern of ventral striatal activity we highlight dovetails with recent data suggesting that ventral striatal responses to financial outcomes reflect a prediction-error signal (PE) rather than a goal value signal (Nieuwenhuis et al., 2005; Yacubian et al., 2006; Hare et al., 2008). To have an efficient estimation of value it is required that the computation of reward magnitude is scaled in relation to a predicted magnitude (Tobler et al., 2005) or that the value of a current event is computed in relation to an event that has not happened yet (Montague and Berns, 2002). More specifically, the ability to calibrate or scale the value signal on a set reference point has been thought to reflect the dynamic by which neuron firing is normalized across inputs (Seymour and McClure, 2008), a dynamic that has been well documented in vision research (Adler et al., 1993).

Whereas activity in ventral striatum robustly correlated with increases in $\Delta$-WTA and decreases in $\Delta \mathrm{WTP}$, insula activity selectively correlated with increases in $\Delta$-WTA (but not decreases in $\Delta$-WTP). This suggests that although the pattern of striatal activity is consistent with a reward prediction error (PE) signal, the pattern of activity in the insula may instead reflect a risk prediction error signal ( $r-P E$ ) (Preuschoff and Bossaerts, 2007) that is referenced to the position of the subject either as seller or buyer. More precisely, when subjects have the opportunity to buy a lottery ticket (i.e., WTP condition) they start from a risk-free condition (i.e., cash endowment) and have the possibility of ending up in a situation of risk (i.e., as a participant in the BDM mechanism). Conversely, in the selling condition, subjects' initial position is inherently risky (i.e., in possession of a lottery ticket) which does not change even if they endeavor to sell the ticket as this
Correlation across subjects

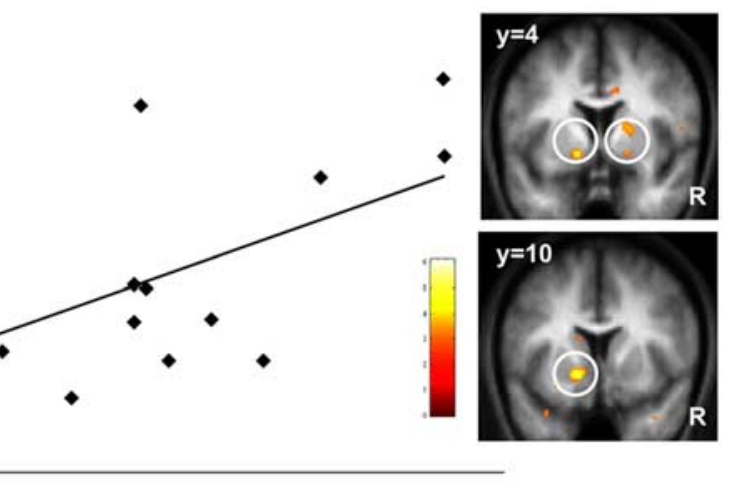

WTA-WTP disparity

5

necessarily also involves a situation of risk (i.e., BDM mechanism). Hence a change in $\mathrm{r}-\mathrm{PE}$ is predicted only in the buying, but not in the selling condition. Our behavioral results provide support for the interpretation that the insula cortex may compute a r-PE that also affects subjects' pricing strategy during the endowment effect task. A closer inspection of the trialby-trial behavioral results (Fig. $2 b$ ) shows that in the selling condition (i.e., WTA, red diamonds in the figure) subjects tend to adopt a more risky price strategy than in the buying condition. In the selling condition, subjects sometimes chose to state a WTA lower than the sub-EV, although they tended in general to state a price higher than the sub-EV (i.e., in line with the endowment effect). In the buying condition, however, subjects very rarely stated a price (i.e., WTP) above the sub$\mathrm{EV}$, consistent with their status-quo risk-free position. As such our results are consistent with a framework wherein the insula encodes an r-PE signal (Preuschoff and Bossaerts, 2007), which may entail a reference-based evaluation of risk during the endowment effect.

It is worth mentioning two relevant recent fMRI studies (Weber et al., 2007; Knutson et al., 2008) that sought to investigate the neural basis of the endowment effect (i.e., the WTA-WTP disparity). It should be noted that neither of these studies elicited a "within subjects" behavioral endowment effect while the subjects were scanned. For example, Knutson and collaborators used a set of consumer goods to estimate a measure of the indifference point for selling and buying at a between subjects level (Knutson et al., 2008). A severe limitation of this design in claiming to speak to the neural basis of endowment effect is the necessity to use indirect estimates, as between subjects or postscanning estimates, of the WTP-WTA disparity.

We argue that our study improves on the design of these previous studies in several respects. First, our within subjects experimental design and choice of fully matched goods in both conditions with a quantifiable expected value (i.e., lottery tickets as opposed to consumer goods) allowed us to directly measure the BOLD correlates with the neural computation 
associated with the WTA-WTP discrepancy during the actual fMRI scanning. On the contrary, indirect estimates that were not obtained at the time of scanning (e.g., "between subjects" or "postscan estimates") (Knutson et al., 2008) used in the previous studies may have little relation with neural computations that the subjects performed at the time of the acquisition of the fMRI signal. In contrast, our study allowed us to identify brain regions in which activity predicts the magnitude of a price discrepancy at actual time of behavioral choice. Second, our experimental design, but not those of Knutson and Weber (Weber et al., 2007; Knutson et al., 2008), included a tightly matched control conditions ("computer"), to ensure that brain activations were specific to the evaluative process, rather than reflective of nonspecific differences engendered between buying and selling.

How value is encoded in the brain remains a key question in neuroscience (Dayan and Abbott, 2001; Montague et al., 2006; Rangel et al., 2008), microeconomics (Glimcher and Rustichini, 2004; Camerer et al., 2005), reinforcement learning (Barto and Sutton, 1998) and animal behavior (Dickinson and Balleine, 1994; Schultz, 2004). From an evolutionary standpoint, it would seem advantageous to maintain a stable representation of a good's value that does not vary as a function of the available alternatives, or the relationship of the decision-maker to the item (cf. endowment effect). Complementary reference-dependent value computations (Koszegi and Rabin, 2006), however, may also have an important adaptive role in the social environment (De Martino et al., 2008), as well as perhaps reflecting inherent constraints of computational neural systems (Seymour and McClure, 2008).

Although much research has been conducted into the neural basis of reference-independent value computations, here we focus on the neural underpinnings of reference-dependent value computations. We show that the OFC and dorsal striatum encode goal decision values, which are not modulated by a subject's role as buyer or seller in the transaction. Critically, we were able to isolate reference dependent prediction errors signals in ventral striatum and insula, which robustly correlated with the magnitude of the behavioral endowment effect. In the future, it will be of considerable interest to investigate how reference-dependent and reference-independent value computations are integrated in the brain to influence behavioral choice.

\section{References}

Adler K, Blakemore C, Pointon M (1993) Vision, coding and efficiency. Cambridge, UK: Cambridge UP.

Balleine BW, Delgado MR, Hikosaka O (2007) The role of the dorsal striatum in reward and decision-making. J Neurosci 27:8161-8165.

Barto AG, Sutton RS (1998) Reinforcment learning: an introduction. Cambridge, MA: MIT.

Becker GM, DeGroot MH, Marschak J (1964) Measuring utility by a singleresponse sequential method. Behav Sci 9:226-232.

Camerer CF, Loewenstein G, Prelec D (2005) Neuroeconomics: why economics needs brains. J Econ Lit XLIII:9-64.

Cromwell HC, Schultz W (2003) Effects of expectations for different reward magnitudes on neuronal activity in primate striatum. J Neurophysiol 89:2823-2838.

Dayan P, Abbott LF (2001) Theoretical neuroscience. Cambridge, MA: MIT.

Delgado MR, Locke HM, Stenger VA, Fiez JA (2003) Dorsal striatum responses to reward and punishment: effects of valence and magnitude manipulations. Cogn Affect Behav Neurosci 3:27-38.

De Martino B, Kumaran D, Seymour B, Dolan RJ (2006) Frames, biases, and rational decision-making in the human brain. Science 313:684-687.
De Martino B, Harrison NA, Knafo S, Bird G, Dolan RJ (2008) Explaining enhanced logical consistency during decision making in autism. J Neurosci 28:10746-10750.

Dickinson A, Balleine B (1994) Motivational control of a goal-directed action. Anim Learn Behav 22:1-18.

Elliott R, Agnew Z, Deakin JF (2008) Medial orbitofrontal cortex codes relative rather than absolute value of financial rewards in humans. Eur J Neurosci 27:2213-2218.

Friston K (1995) Statistical parametric maps in functional imaging: a general linear approach. Hum Brain Mapp 2:189-210.

Glimcher PW, Rustichini A (2004) Neuroeconomics: the consilience of brain and decision. Science 306:447-452.

Hare TA, O’Doherty J, Camerer CF, Schultz W, Rangel A (2008) Dissociating the role of the orbitofrontal cortex and the striatum in the computation of goal values and prediction errors. J Neurosci 28:5623-5630.

Kahneman D, Tversky A (1979) Prospect theory: an analysis of decision under risk. Econometrica 47:263-292.

Kahneman D, Tversky A (2000) Choices, values, and frames. Cambridge, UK: Cambridge UP.

Kahneman D, Knetsch JL, Thaler RH (1990) Experimental tests of the endowment effect and the Coase theorem. J Polit Econ 98:1325-1348.

Kahneman D, Knetsch JL, Thaler RH (1991) Anomalies-the endowment effect, loss aversion, and status-quo bias. J Econ Perspect 5:193-206.

Knutson B, Wimmer GE, Rick S, Hollon NG, Prelec D, Loewenstein G (2008) Neural antecedents of the endowment effect. Neuron $58: 814-822$.

Koszegi B, Rabin M (2006) A model of reference-dependent preferences. Q J Econ 121:1133-1165.

Montague PR, Berns GS (2002) Neural economics and the biological substrates of valuation. Neuron 36:265-284

Montague PR, King-Casas B, Cohen JD (2006) Imaging valuation models in human choice. Annu Rev Neurosci 29:417-448.

Nieuwenhuis S, Heslenfeld DJ, von Geusau NJ, Mars RB, Holroyd CB, Yeung N (2005) Activity in human reward-sensitive brain areas is strongly context dependent. Neuroimage 25:1302-1309.

O’Doherty JP (2004) Reward representations and reward-related learning in the human brain: insights from neuroimaging. Curr Opin Neurobiol 14:769-776.

O'Doherty JP, Deichmann R, Critchley HD, Dolan RJ (2002) Neural responses during anticipation of a primary taste reward. Neuron 33:815-826.

O’Doherty J, Dayan P, Schultz J, Deichmann R, Friston K, Dolan RJ (2004) Dissociable roles of ventral and dorsal striatum in instrumental conditioning. Science 304:452-454.

Padoa-Schioppa C, Assad JA (2006) Neurons in the orbitofrontal cortex encode economic value. Nature 441:223-226.

Padoa-Schioppa C, Assad JA (2008) The representation of economic value in the orbitofrontal cortex is invariant for changes of menu. Nat Neurosci 11:95-102.

Parker MR (2002) Parker's wine buyer's guide, Ed 6. New York: Simon \& Schuster.

Plassmann H, O’Doherty J, Rangel A (2007) Orbitofrontal cortex encodes willingness to pay in everyday economic transactions. J Neurosci 27:9984-9988.

Preuschoff K, Bossaerts P (2007) Adding prediction risk to the theory of reward learning. Ann N Y Acad Sci 1104:135-146.

Rangel A, Camerer C, Montague PR (2008) A framework for studying the neurobiology of value-based decision making. Nat Rev Neurosci 9:545-556.

Roesch MR, Olson CR (2004) Neuronal activity related to reward value and motivation in primate frontal cortex. Science 304:307-310.

Rolls ET, McCabe C, Redoute J (2008) Expected value, reward outcome, and temporal difference error representations in a probabilistic decision task. Cereb Cortex 18:652-663.

Schultz W (2004) Neural coding of basic reward terms of animal learning theory, game theory, microeconomics and behavioural ecology. Curr Opin Neurobiol 14:139-147.

Seymour B, McClure SM (2008) Anchors, scales and the relative coding of value in the brain. Curr Opin Neurobiol 18:173-178.

Seymour B, Daw N, Dayan P, Singer T, Dolan R (2007) Differential en- 
coding of losses and gains in the human striatum. J Neurosci 27:4826-4831.

Tobler PN, Fiorillo CD, Schultz W (2005) Adaptive coding of reward value by dopamine neurons. Science 307:1642-1645.

Tobler PN, Fletcher PC, Bullmore ET, Schultz W (2007) Learning-related human brain activations reflecting individual finances. Neuron 54:167-175.

Tom SM, Fox CR, Trepel C, Poldrack RA (2007) The neural basis of loss aversion in decision-making under risk. Science 315:515-518.

Tremblay L, Schultz W (1999) Relative reward preference in primate orbitofrontal cortex. Nature 398:704-708.

Tricomi EM, Delgado MR, Fiez JA (2004) Modulation of caudate activity by action contingency. Neuron 41:281-292.
Wallis JD (2007) Orbitofrontal cortex and its contribution to decisionmaking. Annu Rev Neurosci 30:31-56.

Wallis JD, Miller EK (2003) Neuronal activity in primate dorsolateral and orbital prefrontal cortex during performance of a reward preference task. Eur J Neurosci 18:2069-2081.

Weber B, Aholt A, Neuhaus C, Trautner P, Elger CE, Teichert T (2007) Neural evidence for reference-dependence in real-market-transactions. Neuroimage 35:441-447.

Willig RD (1976) Consumers surplus without apology. Am Econ Rev 66:589-597.

Yacubian J, Gläscher J, Schroeder K, Sommer T, Braus DF, Büchel C (2006) Dissociable systems for gain- and loss-related value predictions and errors of prediction in the human brain. J Neurosci 26:9530. 